\title{
Reconstruction Strategies for GeMS
}

\author{
Benoit Neichel $^{1, \text { a }}$, Francois Rigaut ${ }^{1}$, Matthieu Bec ${ }^{1}$, and Aurea Garcia-Rissmann ${ }^{1}$ \\ Gemini Observatory, c/o AURA, Casilla 603 La Serena, Chile
}

\begin{abstract}
The Gemini Multi-conjugate adaptive optics System (GeMS) is a facility instrument for the GeminiSouth telescope that can provide near-uniform atmospheric compensation over a 1 arc minute square field of view. To provide diffraction-limited image quality at near IR wavelengths across an extended field-of-view, the system includes 5 laser guide stars, 3 natural guide stars and 3 deformable mirrors optically conjugated at $0,4.5$ and $9 \mathrm{~km}$. By many ways, GeMS represents a unique system to prepare and understand any future wide field AO systems planned for the ELTs. In this paper, we present the different strategies developed for the phase volume estimation and correction relative to the LGS high-order path. We compare the performance obtained by a Least-Square Estimator (LSE) and a Minimum Mean Square Error (MMSE) reconstructor. The MMSE reconstructor is found to improve the performance compared to the classical LSE, particularly when the system is affected by noise. We show the results obtained by realistic simulations as well as the first results obtained in laboratory on CANOPUS, the GeMS AO bench.
\end{abstract}

\section{System Overview}

The Gemini Multi-Conjugate Adaptive Optics System (GeMS) includes 5 Laser Guide Stars (LGS), 3 Natural Guide Stars (NGS) and 3 Deformable Mirrors (DMs) optically conjugated at 0, 4.5 and $9 \mathrm{~km}$. The final phase of integration of all optical components and their integration and tests on the Canopus MCAO Gemini South bench is currently underway. More details on the opto-mechanical integration and calibration procedures are given in two companion papers [1,2]. The 5 LGS-WFS are 16x16 subapertures Shack-Hartmann, working with quad-cells. All 5 WFS are absolutely identical, except for their pointing. They provide a total set of 2040 measurements. The main characteristics of the 5 LGS WFS are summarized in table 1 . The 3 DMs are piezo-stack, for which the influence functions have been carefully calibrated on experimental data [1]. The main characteristics of the DMs are given in Tab. 1. For each DM, some actuators are not seen by the LGS-WFS subapertures and will be controlled in a slaved way, through an extrapolator. This later is computed based on the statistics of the turbulence or using Karhunen-Loeve (KL) modes (see Sect. 2.2). The total number of actuators is 917 including 684 valid (seen by the WFSs).

\section{Wavefront Reconstructors}

Our primary goal is to maximize the Strehl Ratio (SR) all over the one arcmin square field, with as good a homogeneity as possible. Currently, the Real Time Computer (RTC) software can only accommodate one matrix multiply (from 2040 slopes to 684 DM errors, see above). Hence, reconstructors of the type Kalman [3], or POLC [4] with reconstruction of the open loop commands (to apply the correct statistics) can not be implemented. This is unfortunate, but a consequence of the fact the RTC was specified before these techniques emerged. Two reconstructors are then considered in this study: the classical Least-Square Estimator (LSE), and a Minimum Mean Square Error (MMSE) reconstructor. Both are cascaded with a classical integrator. In the following, we assume that the relationship between the 684 valid actuator commands $\mathbf{u}$ and the 2040 slopes $\mathbf{m}$ is linear and can be described by :

$$
\mathbf{m}=\mathbf{D u}
$$

where $\mathbf{D}$ is the system interaction matrix.

\footnotetext{
a e-mail: bneichel@gemini.edu
}

This is an Open Access article distributed under the terms of the Creative Commons Attribution-Noncommercial License, which permits unrestricted use, distribution, and reproduction in any noncommercial medium, provided the original work is properly cited. 
First conference on Adaptive Optics for Extremely Large Telescopes

Table 1. LGS-WFS and DMs characteristics of GeMS.

\begin{tabular}{|c|c|}
\hline WFS location in FoV & $\begin{array}{l}\text { Pointing at LGS located at [0",0”]; [-30”,+30”] } \\
{[\quad[+30 ”,+30 ”] ;[+30 ”,-30 ”] ;[-30 ”,-30 ”]}\end{array}$ \\
\hline Number of valid subapertures & 204 per WFS (total 1020) \\
\hline Sampling frequency & up to $800 \mathrm{~Hz}$ \\
\hline WFS pixel size & $1.38 ”$ \\
\hline WFS RON (measured) & $3.5 \mathrm{e}-$ \\
\hline LGS Spot Size & $1 "$ \\
\hline Altitudes of the DMs & {$[0 \mathrm{~km}, 4.5 \mathrm{~km}, 9 \mathrm{~km}]$} \\
\hline Number of Actuators & [293 (240 valid), 416 (324 valid), 208 (120 valid)] \\
\hline Inter-actuator Coupling & {$[33 \%, 33 \%, 33 \%]$} \\
\hline Physical actuator pitch & {$[5 \mathrm{~mm}, 5 \mathrm{~mm}, 10 \mathrm{~mm}]$} \\
\hline
\end{tabular}

\subsection{Least Square Estimator (LSE)}

In the classical Least Square approach, the actuator commands are derived from the measurements by using a Truncated Singular Value Decomposition (TSVD) generalized inverse of eq. 1. The LSE reconstructor $\mathbf{R}_{\mathbf{L S E}}$ then simply writes as:

$$
\mathbf{R}_{\mathbf{L S E}}=\left(\mathbf{D}^{T} \mathbf{D}\right)^{\dagger} \mathbf{D}^{T}
$$

The main difficulty in such an approach is to decide how many modes should be removed in the inversion. Indeed, the WFS sensitivity to a specific mode need not necessarily correlate with the atmospheric content in that mode. Some modes that are poorly sensed by the WFS may contain a non-negligible amount of atmospheric turbulence statistics. For our system geometry, the "natural" condition number of $\mathbf{D}^{T} \mathbf{D}$ is 410.8 and the distribution of the eigenvalues is plotted on the left of Fig.1. A way to optimize the performance is then to tune the number of truncated modes. Given the low condition number of $\mathbf{D}^{T} \mathbf{D}$ a direct inverse could be considered. However, we do find that with no (or too few) modes truncated, waffle modes appear and make the system unstable. As it is quite difficult to know beforehand how many modes should be truncated to obtain the best performance, we have tested a set of condition number for each configuration (noise level) and chosen the optimal one. Fig.1 (right) show the mean SR computed in a $80 \mathrm{x} 80 \operatorname{arcsec}^{2}$ FoV for different LGS flux and different number of filtered modes (see Sect. 3 for more details on the simulation conditions). As expected, when the system is more noisy, more modes need to be filtered to reach the optimal performance. For each noise configuration, we will use the optimal thresholds as derived form Fig.1. Finally, Fig.3 (left) show the $\mathbf{R}_{\mathbf{L S E}}$ matrix when 120 modes have been filtered.

\subsection{Minimum Mean Square Error (MMSE)}

We use an MMSE reconstructor defined by:

$$
\mathbf{R}_{\mathbf{M M S E}}=\mathbf{C}_{\mathbf{u}} \mathbf{D}\left[\mathbf{D C} \mathbf{C}_{\mathbf{u}} \mathbf{D}^{T}+\alpha \mathbf{C}_{\mathbf{b}}\right]^{-1}
$$

This reconstructor includes prior knowledge of the noise and turbulence statistics through the $\mathbf{C}_{\mathbf{b}}$ (2040x2040) and $\mathbf{C}_{\mathbf{u}}(684 \times 684)$ covariance matrices.

For $\mathbf{C}_{\mathbf{b}}$, we consider the WFS subapertures as statistically independent with respect to noise. The 

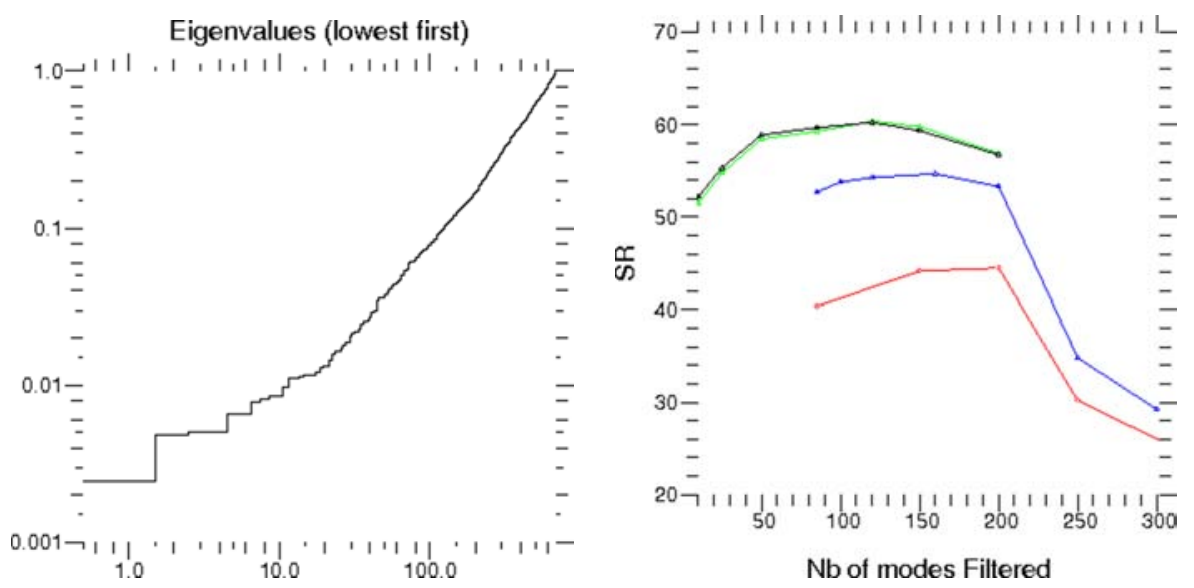

Fig. 1. Left: Eigenvalues distribution for the 3DMs - 5LGS MCAO system. Note that eigenvalues have been normalized to the highest one, and that lowest eigenvalues are plotted first. Right: H-Band SR for typical turbulence conditions as a function of the number of truncated modes in the TSVD for different LGS flux (black:360pht/sub/frame, green:180pht/sub/frame, blue:45pht/sub/frame, red:22pht/sub/frame).

noise covariance is then simply a diagonal matrix with the subaperture noise variance on the diagonal: $\mathbf{C}_{\mathbf{b}}=\sigma_{\text {noise }}^{2} \mathbf{v I}$, where $\mathbf{I}$ is the identity, $\mathbf{v}$ is a weighting factor to account for the partial illumination in the edge apertures and $\sigma_{\text {noise }}^{2}$ is computed from [5] for a quad-cell WFS.

The matrix $\mathbf{C}_{\mathbf{u}}(684 \times 684)$ stands for the covariance matrix of the command vector $\mathbf{u}$. It contains the prior knowledge on the turbulence spatial statistics expressed in the DM space. Because turbulence is uncorelated between layers, it is a block matrix, with as many blocks as DMs. Each block being derived using:

$$
\mathbf{C}_{\mathbf{u}}=\mathbf{P}_{\mathbf{k l 2} \mathbf{u}} \mathbf{C}_{\varphi}\left(\mathbf{P}_{\mathbf{k} \mathbf{2} \mathbf{u}}\right)^{T}
$$

where, $\mathbf{P}_{\mathbf{k l 2} \mathbf{u}}$ is a projection matrix from actuators to modes computed as the optimal basis with respect to the DM influence functions and the turbulence conditions. Fig.2 (right) show the first 3 modes (top) and last 3 modes (bottom) computed for DM1 using this $\mathbf{P}_{\mathbf{k l 2} \mathbf{u}}$ matrix. The matrix $\mathbf{C}_{\varphi}$ is diagonal and it is filled with the variance associated with each of these modes. Fig.2 (left) show the resulting $\mathbf{C}_{\mathbf{u}}$ matrix, Fig.3 (right) show a $\mathbf{R}_{\text {MMSE }}$ matrix.

It is important to note that this MMSE reconstructor will minimize the residual error (and thus maximize the Strehl Ratio) for a system working in open-loop. In particular, the regularizing term $\mathbf{C}_{\mathbf{u}}$ correspond to the statistics of the turbulence for an open-loop system. Applying this reconstructor for a close-loop system is then clearly not optimal. However, as shown by e.g. [6,7], this reconstructor can effectively provide a good performance in close loop operations. Indeed, even if the (statistical) SNR associated with each mode is not exact, a perfect knowledge of the noise priors is not required to obtain an accurate tomographic phase reconstruction [8]. In addition, as introduced in [7], we use an $a d$-hoc parameter ( $\alpha$ in eq.3) that will be adjusted for each SNR case to optimize the performance (see Sect. 3) and assure stability. Finally, note that we have implicitly supposed that the number of turbulence layers that we sought to estimate is equal to the number of DMs, and that the DMs are conjugated to the same altitudes as the turbulence layers. This is the so-called model approximation as introduced by [9]. A more complete reconstruction scheme (e.g. Minimum Variance) should include a reconstruction step on several layers, and then a projection step with an optimization in the field. This reconstructor will be studied in a forthecoming work.

\section{Simulation Results}

The whole MCAO system is studied with a simulation tool called yao, written in yorick by F. Rigaut. This tool closely models all the characteristics of GEMS as it has been calibrated to take into account 

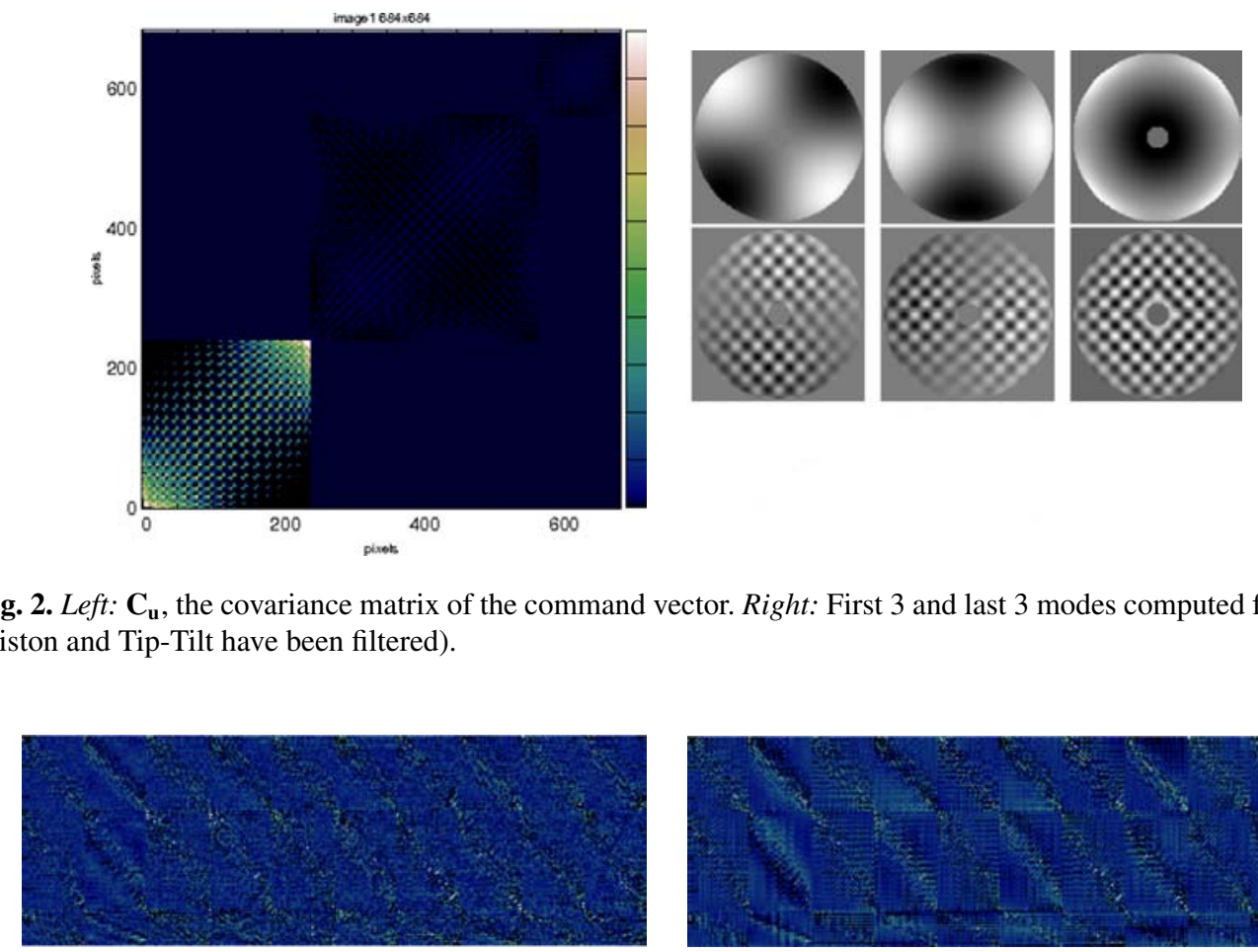

Fig. 2. Left: $\mathbf{C}_{\mathbf{u}}$, the covariance matrix of the command vector. Right: First 3 and last 3 modes computed for DM1 (piston and Tip-Tilt have been filtered).

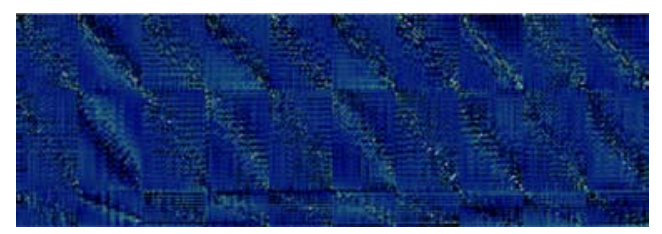

Fig. 3. Left: Command matrix for the LSE reconstructor: $\mathbf{R}_{\mathbf{L S E}}$. Right: Command matrix for the MMSE reconstructor: $\mathbf{R}_{\text {MMSE }}$.

Table 2. Atmospheric parameters used in the simulations.

\begin{tabular}{ccccccc}
\hline Layer number & 1 & 2 & 3 & 4 & 5 & 6 \\
\hline Layer height $(\mathrm{m})$ & 0 & 1800 & 3300 & 5800 & 7400 & 10100 \\
Fraction of $\mathrm{C}_{n}^{2}$ & 0.646 & 0.08 & 0.119 & 0.035 & 0.025 & 0.095 \\
Layer speed $(\mathrm{m} / \mathrm{s})$ & 5 & 7.5 & 12 & 25 & 34 & 21 \\
\hline
\end{tabular}

measurements of the system components, such as the mirror influence functions, actuator gains, WFS subaperture gains, CCD noise, etc... It can be used to compute synthetic control matrices as well [1]. The atmospheric parameters used in the simulation are gathered in Tab.2. They are based on site monitoring data obtained at Cerro Pachon. The total Fried parameter $r_{0}$ is $0.166 \mathrm{~m}$ at $500 \mathrm{~nm}$. In a first approximation, we will assume that the LGS provide a true measurement of the atmospheric Tip-Tilt, and that no NGS are needed. In fact, the NGS low order part is supposed to be orthogonal to the LGS correction, and thus can be considered separately [10]. This assumption will then affect the absolute value of the performance given below, but it should not modify the relative trends. Performance is evaluated in term of SR ( $\mathrm{H}$ band) in a 9x9 grid regularly spaced on a 80"x80" FoV. In Fig.4 we show the long exposure SR map obtained after 10000 simulation iterations for respectively the LSE reconstructor (top row) and the MMSE reconstructor (bottom row). For each reconstructor several LGSWFS flux have been tried, from 22pht/subap/frame to 360pht/subap/frame. For each noise configuration, the optimal threshold for the LSE, and the optimal $\alpha$ parameter have been used (see Fig.4 for their values). SR average and standard deviation are given in the figure caption. The main results of Fig.4 is that the MMSE reconstructor performs consistently better than the LSE when the system is affected by noise, and the differential grows with the amplitude of the noise. Moreover, the performance homogeneity is 

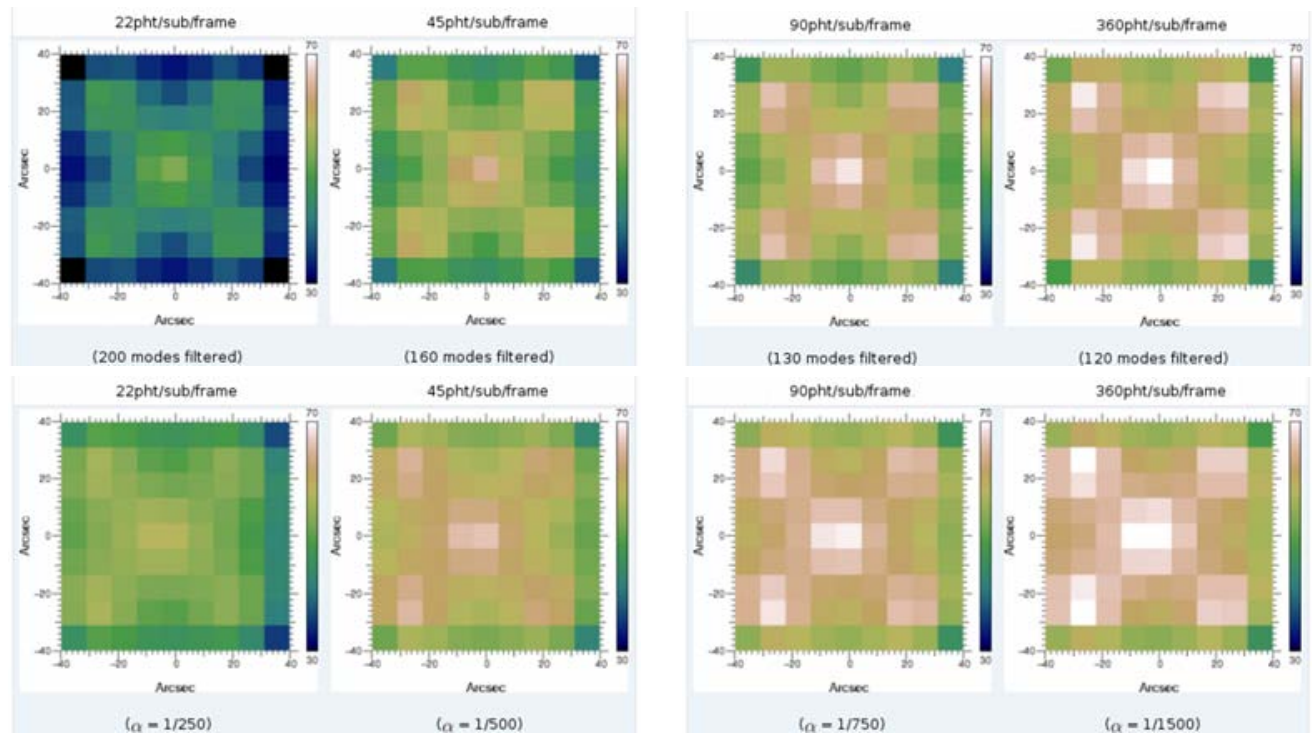

Fig. 4. Top: SR maps for the LSE reconstructor for different flux. Optimal number of filtered modes is also given.

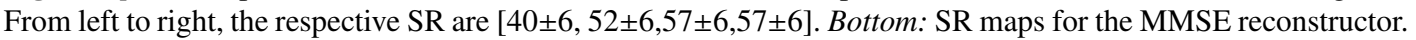
$\mathrm{SR}=[51 \pm 5,58 \pm 5,61 \pm 5,62 \pm 5]$, optimal values for $\alpha$ are given.
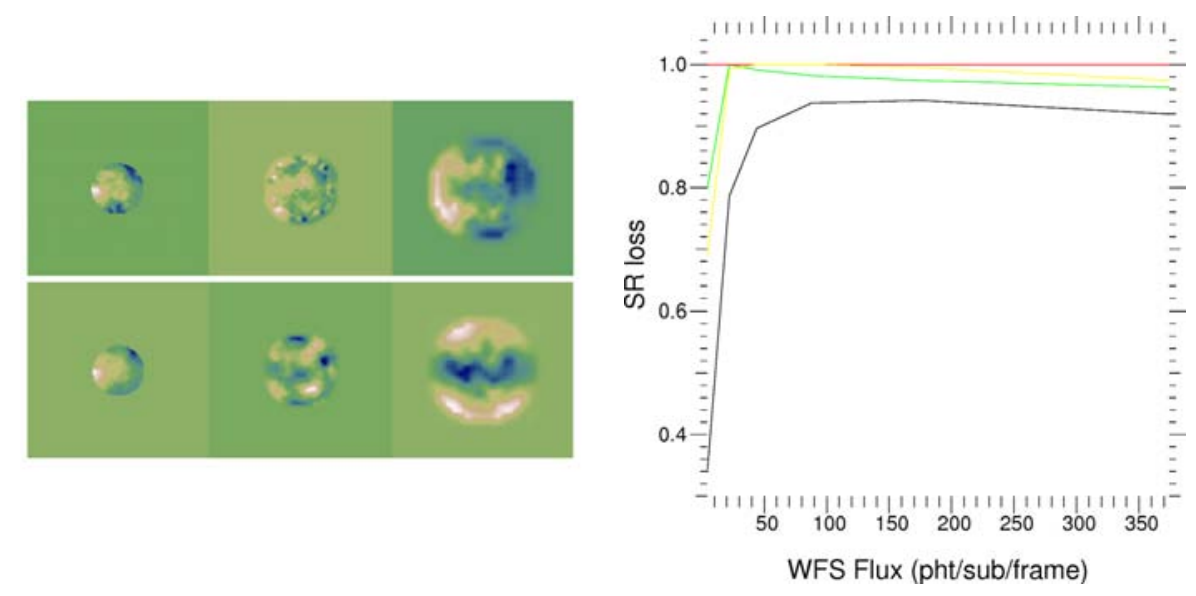

Fig. 5. Left: DM Shape after 10000 iterations for the LSE (top) and MMSE (bottom) reconstructors. Right: SR loss when a wrong $\alpha$ parameter is used : MMSE with optimal alpha (red), MMSE for alpha set to 1/500 (yellow), MMSE for alpha set to $1 / 250$ (green) and LSE (black).

improved with the MMSE reconstructor. In Fig.5 (left) we show the DM shape after 10000 iterations for the LSE (left) and the MMSE (right) reconstructors. As described in [6], the waffle modes present in the LSE matrix are filtered with the MMSE method. This is a consequence of the modal decomposition presented in Fig.2 (right): modes with the lowest SNR are waffle modes. In Fig.5 (right) we evaluate the sensibility of the MMSE performance to the $\alpha$ parameter. The yellow curve show the SR loss if the $\alpha$ parameter is set to 1/500 for all noise configuration (see Fig. 4 for optimal values), the green curve stands for $\alpha$ set to 1/250 and the black curve shows the SR loss when an LSE reconstructor is used. This demonstrates that the performance obtained with the MMSE is not really sensitive to the $\alpha$ parameter and at least always better than the LSE one, even if the $\alpha$ parameter is not optimal. 


\section{Bench Results}

Our capabilities to generate turbulence in the lab is limited as we use the DMs themselves it, following a Von-Karman spectrum up to the cut off frequency of the DMs. In addition, we do not already have an imager in the science focal plane (but it should be installed soon) and the final performance is estimated from the residual slopes on each LGS-WFS. The limitations of this approach are that (1) we can only evaluate the performance on the LGS directions, and (2) the perturbations are perfectly conjugated to the DMs altitudes. Despite these limitations, we have started to compare both reconstructors on the bench, at least to obtain first order results, test the stability and performance under different noise configuration. For high SNR conditions, we find that the performance achieved with the MMSE were as good as for the LSE, as expected from simulations. However, the shape of the DMs appears to be smoother in the MMSE case than in the LSE one, that will probably results in better SR inside the guide stars and less waffle. For lower SNR conditions, the MMSE provides slightly better results than the LSE: 3\% in SR (average over the LGS directions). This is slightly less than expected from simulations, but without a full turbulator generator and an estimation of the performance in the scientific focal plane, it is difficult to do further characterizations.

\section{Conclusion}

We have demonstrated with simulations and on the bench that the use of an MMSE like reconstructor could improve GeMS performance. In continuation of this work, we are currently investigating a full MV reconstructor and a constrained reconstructor as introduced by [11]. On the other hand, we are investigating different temporal filters, like predictive controllers, to evaluate the gain of such control laws. Finally, we have started to investigate whether the RTC could be updated to accommodate a POLC reconstruction, even though this is a remote possibility. All these reconstruction schemes will be tested on the bench, and later on the sky.

The authors wish to thank Thierry Fusco, Jean-Marc Conan, Caroline Kulcsar, Henri-Francois Raynaud, Cyril Petit, Anne Costille, Jean-Pierre Veran, Gerad Rousset, Eric Gendron, Damien Gratadour and Fabrice Vidal for helpful comments, collaborations and fruitful discussions. The Gemini Observatory is operated by the Association of Universities for Research in Astronomy, Inc., under a cooperative agreement with the NSF on behalf of the Gemini partnership: the National Science Foundation (United States), the Science and Technology Facilities Council (United Kingdom), the National Research Council (Canada), CONICYT (Chile), the Australian Research Council (Australia), Ministerio da Ciencia e Tecnologia (Brazil), and Ministerio de Ciencia, Tecnologia e Innovacion Productiva (Argentina).

\section{References}

1. F. Rigaut et al., this conference

2. A. Garcia Rissmann, F. Rigaut, M. Bec, et al., this conference

3. C. Petit, J.M. Conan, C. Kulcsar and H.-F. Raynaud, JOSA A, 26, (2009) 1307-1325

4. L. Gilles, Applied Optics, 44, (2005), 993-1002

5. S. Thomas, T. Fusco, A. Tokovinin, et al. MNRAS, 371, (2006) 323-336

6. M. Le Louarn, MNRAS, 334, (2002) 865-874

7. M. van Dam, D. Le Mignant, B. Macintosh, Applied Optics, 43, (2004) 5458-5467

8. B. Neichel, T. Fusco, J.M. Conan, JOSA A, 26, (2009) 219-231

9. T. Fusco, J.M. Conan, V. Michau, L. Mugnier, G. Rousset, Optics Letter 24, (1999) 1472-1474

10. L. Gilles, B. Ellerbroek, JOSA A, 25, (2008) 2427-2433

11. B. Ellerbroek, JOSA A 11, (1994) 783-805 